\title{
Relativistic symmetries of Schioberg and general Manning-Rosen potentials and the effects of tensor coupling
}

\author{
A. N. Ikot ${ }^{1 *}$, E. Maghsoodi ${ }^{2}$, C. N.Isonguyo ${ }^{1}$, S. Zarrinkamar ${ }^{3}$ and H. Hassanabadi ${ }^{2}$ \\ ${ }^{1}$ Theoretical Physics Group, Department of Physics, University of Uyo-Nigeria \\ ${ }^{2}$ Department of Basic Sciences, Shahrood Branch, Islamic Azad University, \\ Shahrood, Iran \\ ${ }^{3}$ Department of Basic Sciences, Garmsar Branch, Islamic Azad University, \\ Garmsar, Iran
}

Received: October 28, 2013

\begin{abstract}
In this paper we solve the Dirac equation with Schioberg and general ManningRosen potentials including the Coulomb-like tensor interaction. The approximate analytical bound state solutions of the Dirac equation with the Schioberg and Manning-Rosen potential, energy equations and the corresponding unnormalized wave functions are obtained in a closed form using SUSYQM. We have also reported the numerical results to show the effect of the tensor interaction.
\end{abstract}

Key words: Dirac equation, spin symmetry, pseudospin symmetry, Coulomb tensor interaction, Schioberg potential, general Manning-Rosen potential

\section{Introduction}

The concept of SUSYQM has been reported in [1,2], and the references therein provided theoretical physicists with a powerful tool to deal with the non-relativistic Schrödinger equation. However, after some years this powerful tool of mathematical physics was applied to other wave equations in quantum mechanics such as Dirac, Klein-Gordon, DuffinKemmer Petiau (DKP) and spinless Salpeter equations [3-10]. Different methods have been used to deal with Dirac equations with physically motivated potentials. Such methods include Nikiforov-Uvarov (NU) method [11], asymptotic iteration method (AIM) [12], shape invariance [13], super symmetric quantum mechanics (SUSYQM) [1-3,14], factorization method [15] and others [16]. Consequently, the recent search for the solutions of the Dirac equation with physical potential models are expected to lead to the discovery of new phenomena in addition to the spin and pseudospin symmetry discovered many years ago in the nuclei of atom in the Dirac theory [17] in nuclear physics to explain the experimental observation of the quasi-degeneracy in single-nucleon doublets between normal parity orbitals $\left(n, l, j=l+\frac{1}{2}\right)$ and $\left(n-1, l+2, j=l+\frac{3}{2}\right)$ where $n, l$ and $j$ represent

\footnotetext{
${ }^{*}$ Corresponding author

Email address: ndemikotphysics@gmail.com
} 
the radial, orbital and total angular momentum quantum numbers respectively. These relativistic symmetries have been used to form an effective nuclear shell model [18] and to explain other different physical phenomena in nuclear structure such as deformation, super-deformation, magnetic moment, and identical bands [19]. Within the framework of the Dirac theory, spin symmetry occurs when the difference of potential between the time-component of a 4-vector potential, $V(r)$ and Lorentz-scalar potential $S(r)$ is a constant, that is $\Delta(r)=V(r)-S(r)=$ const. On the other hand, the pseudospin symmetry arises when the sum of the potential of the time-component of a 4-vector potential $V(r)$ and the Lorentz-scalar potential $S(r)$ is a constant, that is $\Sigma(r)=V(r)+S(r)=$ const. The concepts of pseudospin and spin symmetries have been investigated on a number of potentials for different potential models [20-21]. Also, the tensor interaction term was introduced into the Dirac equation with the replacement $\vec{p}=\vec{p}-i M \omega \beta \cdot \vec{r} U(r)$ and a spinorbit coupling was added to the Dirac Hamiltonian [22]. Therefore, the effects of tensor interactions in the Dirac equation have become an interesting research area in relativistic quantum mechanics. The introduction of Coulomb-like tensor (CLT) terms has been reported in various sources, to remove the degeneracies in the Dirac theory [23-25]. However, Hassanabadi et al. [26] for the first time also showed that the addition of Yukawa-like tensor (YLT) term besides the Coulomb-like term also removes the degeneracies in the Dirac theory as expected. Recently, we proposed a generalized tensor (GLT) [27] (CLT plus YLT) term in the Dirac equation and this, as a matter of fact, also removes the degeneracies in the Dirac theory.

In this paper we report on the solution of Dirac equation for the combined Schioberg (SC) and general Manning-Rosen (GMR) potentials under the CLT interaction. The SC and GMR potentials are defined as in [28-29],

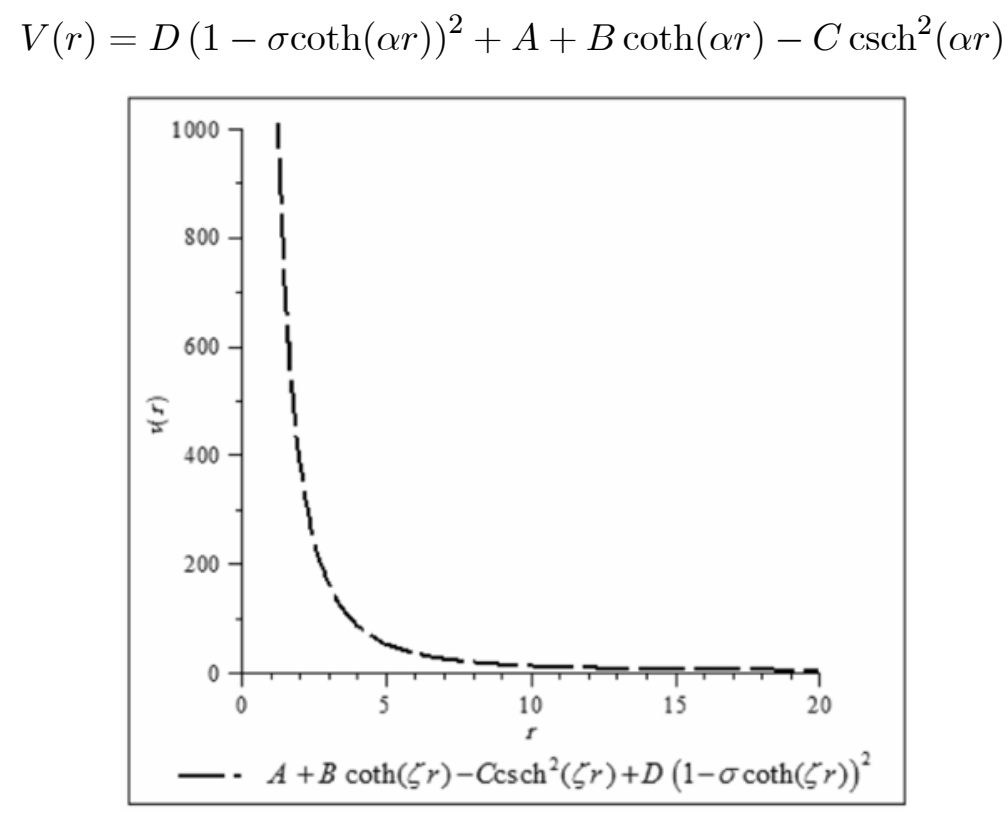

Figure 1. Schematic representation of a focal conic domain confined between two parallel plates.

where $\alpha$ is the screening parameter and $D, A, B$ and $C$ are the coupling strengths of the potential. We have plotted the behavior of the potential in Fig (1). The paper is organized 
as follows. In section 2 we review the SUSYM formalism. The Dirac theory within the framework of spin and pseudospin symmetry with tensor interaction is given in section 3 . Subsections 3.1 and 3.2 are devoted to the solutions of the Dirac equation for new Yukawa potential. The numerical results of our work are presented in section 4 . Finally, we give a brief conclusion in section 5 .

\section{SUSYQM}

We include this short introduction to SUSYQM to proceed on in a continues manner. In SUSUQM we normally deal with the partner Hamiltonians [14]

$$
H_{ \pm}=\frac{p^{2}}{2 m}+V_{ \pm}(x)
$$

where

$$
V_{ \pm}(x)=\Phi^{2}(x) \pm \Phi^{\prime}(x)
$$

In the case of good SUSY, i.e. $E_{0}=0$, the ground state of the system is obtained via

$$
\Phi_{0}^{-}(x)=C e^{-U}
$$

where $C$ is a normalization constant and

$$
U(x)=\int_{x_{0}}^{x} d z \Phi(z)
$$

Next, if the shape invariant condition

$$
V_{+}\left(a_{0}, x\right)=V_{-}\left(a_{1}, x\right)+R\left(a_{1}\right)
$$

where $a_{1}$ is a new set of parameters uniquely determined from the old set $a_{0}$ via the mapping $F: a_{0} \mapsto a_{1}=F\left(a_{0}\right)$ and $R\left(a_{1}\right)$ does not include $x$, the higher state solutions are obtained via

$$
\begin{gathered}
E_{n}=\sum_{s=1}^{n} R\left(a_{s}\right), \\
\Phi_{n}^{-}\left(a_{0}, x\right)=\prod_{s=0}^{n-1}\left(\frac{A^{\dagger}\left(a_{s}\right)}{\left[E_{n}-E_{s}\right]^{1 / 2}}\right) \Phi_{0}^{-}\left(a_{n}, x\right), \\
\Phi_{n}^{-}\left(a_{n}, x\right)=C \exp \left\{-\int_{0}^{x} d z \Phi\left(a_{n}, z\right)\right\},
\end{gathered}
$$

where

$$
A_{s}^{\dagger}=-\frac{\partial}{\partial x}+\Phi\left(a_{s}, x\right)
$$

Therefore, this condition determines the spectrum of the bound states of the Hamiltonian

$$
H_{s}=-\frac{\partial^{2}}{\partial x^{2}}+V_{-}\left(a_{s}, x\right)+E_{s} .
$$

and the energy eigenfunctions of 


$$
H_{s} \Phi_{n-s}^{-}\left(a_{s}, x\right)=E_{n} \Phi_{n-s}^{-}\left(a_{s}, x\right), \quad n \geq s
$$

are related via $[4,21]$

$$
\Phi_{n-s}^{-}\left(a_{s}, x\right)=\frac{A^{\dagger}}{\left[E_{n}-E_{s}\right]^{1 / 2}} \Phi_{n-(s+1)}^{-}\left(a_{s+1}, x\right)
$$

\section{Dirac Equation with a Tensor Coupling}

The Dirac time-independent equation with a tensor potential $U(r)$ in the relativistic unit $(\hbar=c=1)$ is written as [23-27]:

$$
[\vec{\alpha} \cdot \vec{p}+\beta(M+S(r))-i \beta \vec{\alpha} \cdot \hat{r} U(r)] \Psi(r)=[E-I V(r)] \Psi(r),
$$

where $E$ is the relativistic energy of the system, $\vec{p}=-i \vec{\nabla}$ is the three-dimensional momentum operator and $M$ is the mass of the fermionic particle. $\vec{\alpha}, \beta$ are the $4 \times 4$ Dirac matrices given as

$$
\vec{\alpha}=\left(\begin{array}{cc}
0 & \vec{\sigma}_{i} \\
\vec{\sigma}_{i} & 0
\end{array}\right), \quad \beta=\left(\begin{array}{cc}
I & 0 \\
0 & -I
\end{array}\right)
$$

where $I$ is a $2 \times 2$ unit matrix and $\vec{\sigma}_{i}$ are the Pauli three-vector matrices defined as

$$
\vec{\sigma}_{1}=\left(\begin{array}{cc}
0 & 1 \\
1 & 0
\end{array}\right), \quad \sigma_{2}=\left(\begin{array}{cc}
0 & -i \\
i & 0
\end{array}\right), \quad \sigma_{3}=\left(\begin{array}{cc}
1 & 0 \\
0 & -1
\end{array}\right)
$$

The eigenvalues of the spin-orbit coupling operator are $\kappa=\left(j+\frac{1}{2}\right)>0, \kappa=-\left(j+\frac{1}{2}\right)<$ 0 for unaligned $j=l-\frac{1}{2}$ and aligned spin $j=l+\frac{1}{2}$ cases, respectively. The set $\left(H, K, J^{2}, J_{z}\right)$ forms a complete set of conserved quantities. Thus, we can write the spinors as $[23-27]$

$$
\vec{\Psi}_{n \kappa}(r)=\frac{1}{r}\left(\begin{array}{cc}
F_{n \kappa}(r) & Y_{j m}^{l}(\theta, \varphi) \\
i G_{n \kappa}(r) & Y_{j m}^{l}(\theta, \varphi)
\end{array}\right)
$$

where $F_{n \kappa}(r)$ and $G_{n \kappa}(r)$ represent the upper and lower components of the Dirac spinors. $Y_{j m}^{l}(\theta, \varphi), Y_{j m}^{\tilde{l}}(\theta, \varphi)$ are the spin and pseudospin spherical harmonics and $m$ is the projection on the z-axis. With the other known identities [23-27]

$$
\begin{aligned}
& (\vec{\sigma} \cdot \vec{A})(\vec{\sigma} \cdot \vec{B})=\vec{A} \cdot \vec{B}+i \vec{\sigma} \cdot(\vec{A} \times \vec{B}), \\
& \vec{\sigma} \cdot \vec{p}=\vec{\sigma} \cdot \vec{r}\left(\vec{r} \cdot \vec{p}+i \frac{\vec{\sigma} \cdot \vec{L}}{r}\right)
\end{aligned}
$$

as well as 


$$
\begin{aligned}
& (\vec{\sigma} \cdot \vec{L}) Y_{j m}^{\tilde{l}}(\theta, \varphi)=(\kappa-1) Y_{j m}^{\tilde{l}}(\theta, \varphi) \\
& (\vec{\sigma} \cdot \vec{L}) Y_{j m}^{l}(\theta, \varphi)=-(\kappa-1) Y_{j m}^{l}(\theta, \varphi) \\
& (\vec{\sigma} \cdot \vec{r}) Y_{j m}^{l}(\theta, \varphi)=-Y_{j m}^{\tilde{l}}(\theta, \varphi) \\
& (\vec{\sigma} \cdot \vec{r}) Y_{j m}^{\tilde{l}}(\theta, \varphi)=-Y_{j m}^{l}(\theta, \varphi)
\end{aligned}
$$

we obtain the coupled equations [23-27],

$$
\begin{aligned}
& \left(\frac{d}{d r}+\frac{\kappa}{r}-U(r)\right) F_{n \kappa}=\left(M+E_{n \kappa}-\Delta(r)\right) G_{n \kappa}(r), \\
& \left(\frac{d}{d r}+-\frac{\kappa}{r}+U(r)\right) G_{n \kappa}=\left(M-E_{n \kappa}+\Sigma(r)\right) F_{n \kappa}(r),
\end{aligned}
$$

where,

$$
\begin{aligned}
& \Delta(r)=V(r)-S(r), \\
& \Sigma(r)=V(r)+S(r) .
\end{aligned}
$$

Eliminating $F_{n \kappa}(r)$ and $G_{n \kappa}(r)$ in favor of each other in Eqs. (18) and (19), we obtain the second-order Schrödinger-like equations

$$
\begin{aligned}
& \left\{\frac{d^{2}}{d r^{2}}-\frac{\kappa(\kappa+1)}{r^{2}}+\frac{2 \kappa U(r)}{r}-\frac{d U(r)}{d r}-U^{2}(r)-\left(M+E_{n \kappa}-\Delta(r)\right)\left(M-E_{n \kappa}+\Sigma(r)\right)+\right. \\
& \left.+\frac{\frac{d \Delta(r)}{d r}\left(\frac{d}{d r}+\frac{\kappa}{r}-U(r)\right)}{\left(M+E_{n \kappa}-\Delta(r)\right.}\right\} F_{n \kappa}(r)=0, \\
& \left\{\frac{d^{2}}{d r^{2}}-\frac{\kappa(\kappa-1)}{r^{2}}+\frac{2 \kappa U(r)}{r}+\frac{d U(r)}{d r}-U^{2}(r)-\left(M+E_{n \kappa}-\Delta(r)\right)\left(M-E_{n \kappa}+\Sigma(r)\right)+\right. \\
& \left.+\frac{\frac{d \Sigma(r)}{d r}\left(\frac{d}{d r}-\frac{\kappa}{r}+U(r)\right)}{\left(M-E_{n \kappa}+\Sigma(r)\right.}\right\} G_{n \kappa}(r)=0,
\end{aligned}
$$

with $\kappa(\kappa-1)=\tilde{l}(\tilde{l}+1), \kappa(\kappa+1)=l(l+1)$.

\subsection{Pseudospin Symmetry Limit under CLT}

In the pseudospin symmetry limit, $\frac{d \Sigma(r)}{d r}=0$ or $\Sigma(r)=C_{p s}=$ const. [17-19]. Here, we intend to study the SC and GMR potentials (which is plotted in Fig. 1)

$$
\Delta(r)=A^{p s}+B^{p s} \operatorname{coth}(\zeta r)-C^{p s} \operatorname{csch}^{2}(\zeta r)+D^{p s}(1-\sigma \operatorname{coth}(\zeta r))^{2},
$$

besides the Coulomb tensor interaction [23] 


$$
U(r)=-\frac{H_{c}}{r} ; \quad r \geq R_{e}
$$

where

$$
H_{c}=\frac{Z_{a} Z_{b} e^{2}}{4 \pi \varepsilon_{0}},
$$

where $R_{e}$ is the Coulomb radius, $Z_{a}, Z_{b}$ denote the charges of the projectile $a$ and the target nuclei $b$ [23]. Substituting Eqs. (24) and (25) into Eq..(23), we get,

$$
\begin{aligned}
& \left\{\frac{d^{2}}{d r^{2}}-\frac{\kappa(\kappa-1)}{r^{2}}-\frac{2 \kappa H_{c}}{r^{2}}+\frac{H_{c}}{r^{2}}-\frac{H_{c}^{2}}{r^{2}}+\left(M-E_{n \kappa}^{p s}+C_{p s}\right)\left[A^{p s}+\right.\right. \\
& \left.\left.+B^{p s} \operatorname{coth}(\zeta r)-C^{p s} \operatorname{csch}^{2}(\zeta r)+D^{p s}(1-\sigma \operatorname{coth}(\zeta r))^{2}\right]\right\} G_{n \kappa}^{p s}= \\
& =\left(M+E_{n \kappa}^{p s}\right)\left(M-E_{n \kappa}^{p s}+C_{p s}\right) G_{n \kappa}^{p s}
\end{aligned}
$$

The corresponding equation is not solvable in an exact way. Consequently, to provide an analytic solution, we have to proceed on an approximate basis. Therefore, we introduce the approximations [30]

$$
\frac{1}{r^{2}} \approx \frac{\zeta^{2}}{\sinh ^{2}(\zeta r)}=\zeta^{2} \operatorname{csch}^{2}(\zeta r),
$$

Substituting Eq. (28) into Eq. (27) yields

$$
\begin{aligned}
& \left\{\frac{d^{2}}{d r^{2}}-\zeta^{2} \eta_{\kappa}\left(\eta_{\kappa}-1\right) \operatorname{csch}^{2}(\zeta r)+\left(M-E_{n \kappa}^{p s}+C_{p s}\right)\left[A^{p s}+B^{p s} \operatorname{coth}(\zeta r)-\right.\right. \\
& \left.\left.-C^{p s} \operatorname{csch}^{2}(\zeta r)+D^{p s}\left(1-2 \sigma \operatorname{coth}(\zeta r)+\sigma^{2} \operatorname{coth}^{2}(\zeta r)\right)\right]\right\} G_{n \kappa}^{p s}= \\
& =\left(M+E_{n \kappa}\right)\left(M-E_{n \kappa}+C_{p s}\right) G_{n \kappa}^{p s},
\end{aligned}
$$

or we rewrite Eq. (29) as,

$$
-\frac{d^{2} G_{n \kappa}^{p s}}{d r^{2}}+V_{\mathrm{eff}}(r) G_{n \kappa}^{p s}=\tilde{E}_{n \kappa}^{p s} G_{n \kappa}^{p s},
$$

where,

$$
\begin{gathered}
V_{\mathrm{eff}}=\tilde{V}_{1 p s} \operatorname{csch}^{2}(\zeta r)+\tilde{V}_{2 p s} \operatorname{coth}(\zeta r) \\
\tilde{E}_{n \kappa}^{p s}=-M^{2}-M C_{p s}+\left(E_{n \kappa}^{p s}\right)^{2}-E_{n \kappa}^{p s} C_{p s}+\left(A^{p s}+D^{p s}+D^{p s} \sigma^{2}\right)\left(M-E_{n \kappa}^{p s}+C_{p s}\right), \\
V_{1 p s}=\zeta^{2} \eta_{\kappa}\left(\eta_{\kappa}-1\right)+M C^{p s}-E_{n \kappa}^{p s} C^{p s}+C^{p s} C_{p s}-M D^{p s} \sigma^{2}+E_{n \kappa}^{p s} D^{p s} \sigma^{2}-C_{p s} D^{p s} \sigma^{2}, \\
V_{2 p s}=2 M \sigma D^{p s}-2 E_{n \kappa}^{p s} \sigma D^{p s}+2 C_{p s} \sigma D^{p s}-M B^{p s}+E_{n \kappa}^{p s} B^{p s}-C_{p s} B^{p s}
\end{gathered}
$$




$$
\begin{aligned}
& \left(H_{c}^{2}+2 \kappa H_{c}-H_{c}+\kappa(\kappa-1)\right)=\left(\kappa+H_{c}\right)\left(\kappa+H_{c}-1\right)=\eta_{\kappa}\left(\eta_{\kappa}-1\right) \rightarrow \\
& \rightarrow \eta_{\kappa}=\left(\kappa+H_{c}\right)
\end{aligned}
$$

In the SUSYQM formalism, the ground state wave function for the lower component is given as,

$$
G_{0, \kappa}^{p s}=\exp \left(-\int \phi(r) d r\right)
$$

This ground state is related to the nonlinear Riccati equation defined as,

$$
\phi^{2}-\phi^{\prime}=V_{\mathrm{eff}}(r)-\tilde{E}_{0, \kappa}^{p s}
$$

for which we proposed a superpotential of the form,

$$
\phi^{p s}=g^{p s}-f^{p s} \operatorname{coth}(\zeta r)
$$

Now, using this superpotential and the expression for the effective potential, the Riccati equation (37) turns into an exact parameter of our study as,

$$
\begin{aligned}
& \left(f^{p s}\right)^{2}+\left(g^{p s}\right)^{2}+\left(f^{p s}\right)^{2} \operatorname{csch}^{2}(\zeta r)-2 f^{p s} g^{p s} \operatorname{coth}(\zeta r)-\zeta f^{p s} \operatorname{csch}^{2}(\zeta r)= \\
& =\tilde{V}_{1 p s} \operatorname{csch}^{2}(\zeta r)+\tilde{V}_{2 p s} \operatorname{coth}(\zeta r)-\tilde{E}_{0, \kappa}^{p s}
\end{aligned}
$$

Or solving equation (39) completely yields,

$$
\begin{gathered}
\tilde{E}_{0, \kappa}^{p s}=-\left(\left(f^{p s}\right)^{2}+\left(g^{p s}\right)^{2}\right), \\
f^{p s}=\frac{\zeta-\sqrt{\zeta^{2}+4 \tilde{V}_{1 p s}}}{2} \\
g^{p s}=-\frac{V_{2 p s}}{2 f^{p s}}
\end{gathered}
$$

Using Eq. (38), we can construct the partner Hamiltonian as,

$$
\begin{aligned}
& V_{\text {eff }+}(r)=\phi^{2}+\frac{d \phi}{d r}=f^{p s}\left(f^{p s}+\zeta\right) \operatorname{csch}^{2}(\zeta r)+\tilde{V}_{2 p s} \operatorname{coth}(\zeta r)+\left(f^{p s}\right)^{2}+\frac{\tilde{V}_{2 p s}^{2}}{4\left(f^{p s}\right)^{2}} \\
& V_{\text {eff- }}(r)=\phi^{2}-\frac{d \phi}{d r}=f^{p s}\left(f^{p s}-\zeta\right) \operatorname{csch}^{2}(\zeta r)+\tilde{V}_{2 p s} \operatorname{coth}(\zeta r)+\left(f^{p s}\right)^{2}+\frac{\tilde{V}_{2 p s}^{2}}{4\left(f^{p s}\right)^{2}}
\end{aligned}
$$

Thus, it is not difficult to show that $V_{+}(r)$ and $V_{-}(r)$ are shape-invariant, i.e.

$$
V_{+}\left(r, \rho_{0}\right)=V_{-}\left(r, \rho_{i}\right)+R\left(\rho_{i}\right)
$$

where $\rho_{0}=f^{p s}$ and $\rho_{i}$ is a function of $\rho_{0}$, i.e., $\rho_{1}=f\left(\rho_{0}\right)=\rho_{0}+\zeta$. Therefore, $\rho_{n}=$ $f\left(\rho_{0}\right)=\rho_{0}+n \zeta$. Consequently, the shape invariance holds via the mapping $f^{p s} \rightarrow f^{p s}+\zeta$. Thus, from Eq. (45), we obtained 


$$
\begin{gathered}
R\left(\rho_{1}\right)=\left(\rho_{0}^{2}+\frac{\tilde{V}_{2 p s}^{2}}{4 \rho_{0}^{2}}\right)-\left(\rho_{1}^{2}+\frac{\tilde{V}_{2 p s}^{2}}{4 \rho_{1}^{2}}\right) \\
R\left(\rho_{2}\right)=\left(\rho_{1}^{2}+\frac{\tilde{V}_{2 p s}^{2}}{4 \rho_{1}^{2}}\right)-\left(\rho_{2}^{2}+\frac{\tilde{V}_{2 p s}^{2}}{4 \rho_{2}^{2}}\right) \\
\cdot \\
\cdot \\
R\left(\rho_{n}\right)=\left(\rho_{n-1}^{2}+\frac{\tilde{V}_{2 p s}^{2}}{4 \rho_{n-1}^{2}}\right)-\left(\rho_{n}^{2}+\frac{\tilde{V}_{2 p s}^{2}}{4 \rho_{n}^{2}}\right) \\
\tilde{E}_{0, \kappa}^{-}=0
\end{gathered}
$$

Therefore, the energy eigenvalues can be obtained from Eq. (46) as,

$$
\tilde{E}_{n \kappa}^{p s-}=\sum_{k=1}^{n} R\left(\rho_{k}\right)=\left(\rho_{0}^{2}+\frac{\tilde{V}_{2 p s}^{2}}{4 \rho_{0}^{2}}\right)-\left(\rho_{n}^{2}+\frac{\tilde{V}_{2 p s}^{2}}{4 \rho_{n}^{2}}\right)
$$

but

$$
\tilde{E}_{n \kappa}^{p s}=\tilde{E}_{n \kappa}^{p s-}+\tilde{E}_{0, \kappa}^{p s}=-\left(\rho_{n}^{2}+\frac{\tilde{V}_{2 p s}^{2}}{4 \rho_{n}^{2}}\right)
$$

with the aid of Eqs. (30-33), (39) and (49), we obtain the energy eigenvalues for the Schioberg and general Manning-Rosen potential for the pseudospin symmetry for any spin orbit quantum number as,

$$
\begin{aligned}
& -M^{2}-M C_{p s}+\left(E_{n \kappa}^{p s}\right)^{2}-E_{n \kappa}^{p s} C_{p s}+\left(A^{p s}+D^{p s}+D^{p s} \sigma^{2}\right)\left(M-E_{n \kappa}^{p s}+C_{p s}\right)+ \\
& +\left(\rho_{n}^{2}+\frac{\tilde{V}_{2 p s}^{2}}{4 \rho_{n}^{2}}\right)=0
\end{aligned}
$$

where

$$
\rho_{n}=\zeta\left(n+\omega^{p s}\right),
$$

$\omega_{p s}=\frac{1}{2}(1 \pm$

$$
\left.\pm \sqrt{1+\frac{4}{\zeta^{2}}\left(\zeta^{2} \eta_{\kappa}\left(\eta_{\kappa}-1\right)+M C^{p s}-E_{n \kappa}^{p s} C^{p s}+C^{p s} C_{p s}-M D^{p s} \sigma^{2}+E_{n \kappa}^{p s} D^{p s} \sigma^{2}-C_{p s} D^{p s} \sigma^{2}\right)}\right)
$$

For the completeness of the description of the potential model under investigation, let us find the corresponding wave functions for the pseudospin symmetry limit. By using the transformation, $z=\operatorname{coth}(\beta r)$, we obtain the lower component of the wave function as, 


$$
\begin{aligned}
& G_{n \kappa}^{p s}(r)=N_{n \kappa}\left(\frac{1}{2}+\frac{\operatorname{coth}(\zeta r)}{2}\right)^{\frac{3}{2}+\sqrt{\frac{9}{4}+\chi_{3}^{p s}}}\left(\frac{1}{2}-\frac{\operatorname{coth}(\zeta r)}{2}\right)^{-\frac{1}{2}+\sqrt{\frac{1}{4}+\chi_{1}^{p s}+\chi_{3}^{p s}-\chi_{2}^{p s}}} \\
& \times_{2} F_{1}\left(-n ; n+1+2 \sqrt{\frac{9}{4}+\chi_{3}^{p s}}+2 \sqrt{\frac{1}{4}+\chi_{1}^{p s}+\chi_{3}^{p s}-\chi_{2}^{p s}} ; 2 \sqrt{\frac{9}{4}+\chi_{3}^{p s}}+1 ;-\operatorname{coth}(\zeta r)\right)
\end{aligned}
$$

where,

$$
\begin{gathered}
\chi_{1}^{p s}=\frac{\tilde{V}_{1 p s}}{\zeta^{2}}, \\
\chi_{2}^{p s}=\frac{\tilde{V}_{1 p s}}{\zeta^{2}}-\frac{\tilde{V}_{2 p s}}{2 \zeta^{2}}, \\
\chi_{3}^{p s}=-\frac{\tilde{V}_{2 p s}}{4 \zeta^{2}}-\frac{\tilde{E}_{n \kappa}^{p s}}{4 \zeta^{2}},
\end{gathered}
$$

On the other hand, the upper component can be found by using the following relation:

$$
F_{n \kappa}^{p s}(r)=\frac{1}{M-E_{n \kappa}^{p s}+C_{p s}}\left(\frac{d}{d r}-\frac{\kappa}{r}+U(r)\right) G_{n \kappa}^{p s}(r) .
$$

\subsection{Spin Symmetry Limit}

In the spin symmetry limit $\frac{d \Delta(r)}{d r}=0$ or $\Delta(r)=C_{s}=$ const. [17-19]. As in the previous section, we consider the sum of the potential and Yukawa tensor interaction as,

$$
\Sigma(r)=A^{s}+B^{s} \operatorname{coth}(\zeta r)-C^{s} \operatorname{csch}^{2}(\zeta r)+D^{s}(1-\sigma \operatorname{coth}(\zeta r))^{2} .
$$

Substituting Eqs. (56) and (25) into Eq. (22) gives,

$$
\begin{aligned}
& \left\{\frac{d^{2}}{d r^{2}}-\frac{\kappa(\kappa+1)}{r^{2}}-\frac{2 \kappa H_{c}}{r^{2}}-\frac{H_{c}}{r^{2}}-\frac{H_{c}^{2}}{r^{2}}-\left(M+E_{n \kappa}-C_{s}\right)\left[A^{s}+B^{s} \operatorname{coth}(\zeta r)-\right.\right. \\
& \left.\left.-C^{s} \operatorname{csch}^{2}(\zeta r)+D^{s}(1-\sigma \operatorname{coth}(\zeta r))^{2}\right]\right\} F_{n \kappa}=\left(M+E_{n \kappa}-C_{s}\right)\left(M-E_{n \kappa}\right) F_{n \kappa}(r)
\end{aligned}
$$

By using approximations (28) into Eq. (58) we obtain:

$$
-\frac{d^{2} F_{n \kappa}}{d r^{2}}+V_{\mathrm{eff}}(r) F_{n \kappa}=\tilde{E}_{n \kappa}^{s} F_{n \kappa}(r)
$$

where,

$$
\begin{gathered}
V_{\mathrm{eff}}(r)=\tilde{V}_{1 s} \operatorname{csch}^{2}(\zeta r)+\tilde{V}_{2 s} \operatorname{coth}(\zeta r), \\
\tilde{E}_{n \kappa}=-M^{2}+\left(E_{n \kappa}^{s}\right)^{2}+M C_{s}-C_{s} E_{n \kappa}^{s}-\left(A^{s}+D^{s}+D^{s} \sigma^{2}\right)\left(M+E_{n \kappa}^{s}-C_{s}\right),
\end{gathered}
$$




$$
\begin{gathered}
\tilde{V}_{1 s}=\zeta^{2} \Lambda_{\kappa}\left(\Lambda_{\kappa}-1\right)-M C^{s}-E_{n \kappa}^{s} C^{s}+C_{s} C^{s}+M D^{s} \sigma^{2}+E_{n \kappa}^{s} D^{s} \sigma^{2}-C_{s} D^{s} \sigma^{2} \\
\tilde{V}_{2 s}=M B^{s}+E_{n \kappa}^{s} B^{s}-C_{s} B^{s}-2 M \sigma D^{s}-2 E_{n \kappa}^{s} \sigma D^{s}+2 C_{s} \sigma D^{s} \\
\left(H_{c}^{2}+2 \kappa H_{c}+H_{c}+\kappa(\kappa+1)\right)=\left(\kappa+H_{c}\right)\left(\kappa+H_{c}+1\right)=\Lambda_{\kappa}\left(\Lambda_{\kappa}-1\right) \rightarrow \\
\rightarrow \Lambda_{\kappa}=\left(\kappa+H_{c}+1\right)
\end{gathered}
$$

Following the same procedures as in the previous section, we find the energy eigenvalues equation of the Dirac theory for the Schioberg and general Manning-Rosen potential in the presence of Coulomb-like tensor interaction as follows:

$$
-M^{2}+\left(E_{n \kappa}^{s}\right)^{2}+M C_{s}-C_{s} E_{n \kappa}^{s}-\left(A^{s}+D^{s}+D^{s} \sigma^{2}\right)\left(M+E_{n \kappa}^{s}-C_{s}\right)+\left(\rho_{n}^{2}+\frac{\tilde{V}_{2 s}^{2}}{4 \rho_{n}^{2}}\right)=0
$$

where

$$
\begin{aligned}
& \rho_{n}=\zeta\left(n+\omega^{s}\right), \\
& \omega_{s}=\frac{1}{2}(1 \pm \\
& \left. \pm \sqrt{1+\frac{4}{\zeta^{2}}\left(\zeta^{2} \Lambda_{\kappa}\left(\Lambda_{\kappa}-1\right)-M C^{s}-E_{n \kappa}^{s} C^{s}+C_{s} C^{s}+M D^{s} \sigma^{2}+E_{n \kappa}^{s} D^{s} \sigma^{2}-C_{s} D^{s} \sigma^{2}\right)}\right)
\end{aligned}
$$

The upper component of the wave function is obtained as follows:

$$
\begin{aligned}
& F_{n \kappa}^{s}(r)=N_{n \kappa}\left(\frac{1}{2}+\frac{\operatorname{coth}(\zeta r)}{2}\right)^{\frac{3}{2}+\sqrt{\frac{9}{4}+\chi_{3}^{s}}}\left(\frac{1}{2}-\frac{\operatorname{coth}(\zeta r)}{2}\right)^{-\frac{1}{2}+\sqrt{\frac{1}{4}+\chi_{1}^{s}+\chi_{3}^{s}-\chi_{2}^{s}}} \\
& \times_{2} F_{1}\left(-n ; n+1+2 \sqrt{\frac{9}{4}+\chi_{3}^{s}}+2 \sqrt{\frac{1}{4}+\chi_{1}^{s}+\chi_{3}^{s}-\chi_{2}^{s}} ; 2 \sqrt{\frac{9}{4}+\chi_{3}^{s}}+1 ;-\operatorname{coth}(\zeta r)\right)
\end{aligned}
$$

where

$$
\begin{gathered}
\chi_{1}^{s}=\frac{\tilde{V}_{1 s}}{\zeta^{2}}, \\
\chi_{2}^{s}=\frac{\tilde{V}_{1 s}}{\zeta^{2}}-\frac{\tilde{V}_{2 s}}{2 \zeta^{2}}, \\
\chi_{3}^{s}=-\frac{\tilde{V}_{2 s}}{4 \zeta^{2}}-\frac{\tilde{E}_{n \kappa}^{s}}{4 \zeta^{2}},
\end{gathered}
$$

and the other component can be simply found via

$$
G_{n \kappa}^{s}(r)=\frac{1}{M+E_{n \kappa}-C_{s}}\left(\frac{d}{d r}+\frac{\kappa}{r}-U(r)\right) F_{n \kappa}^{s}(r)
$$




\section{Numerical Results}

We investigate the energy eigenvalues in the absence $\left(H_{c}=0\right)$ and the presence $\left(H_{c}=\right.$ $0.5)$ and $\left(H_{c}=1\right)$ of the Coulomb-like tensor potential for various values of the quantum numbers $n$ and $\kappa$. The results are reported in Tables 1 and 2 for the Schioberg and general Manning-Rosen potentials under the condition of the pseudospin and spin symmetries, respectively. It can be seen from Tables 1 and 2 that all degeneracies between two states in the spin and pseudospin doublets are removed by tensor interaction $\left(H_{c} \neq 0\right)$. In order to test the accuracy of our work, we have computed some numerical results that are given in Tables 1 and 2, where we used the parameter values as $\zeta=0.05, M=1 \mathrm{fm}^{-1}$, $A^{p s}=-8.75, B^{p s}=8.75, C^{p s}=4.5, C_{p s}=0, D^{p s}=0, \sigma=0$ for the pseudospin symmetry case and $\zeta=0.05, M=1 \mathrm{fm}^{-1}, A^{s}=8, B^{s}=-8, C^{s}=-4.5, C_{s}=0, D^{s}=0$, $\sigma=0$ for the spin symmetry case, and obtained the same results as in [23].

Table 1. Energies in the Pseudospin Symmetry Limit for $\zeta=0.05, M=1 \mathrm{fm}^{-1}$, $A^{p s}=-8.75, B^{p s}=8.75, C^{p s}=4.5, C_{p s}=0, D^{p s}=-2, \sigma=-0.2$.

\begin{tabular}{cccccccc}
\hline \hline & & & & & $E_{n \kappa}^{p s}\left(H_{c}=0 D^{p s}=0\right)$ \\
$\tilde{\ell}$ & $n$ & $\kappa$ & $(\ell, j)$ & $E_{n \kappa}^{p s}\left(H_{c}=0\right)$ & $E_{n \kappa}^{p s}\left(H_{c}=0.5\right)$ & $E_{n \kappa}^{p s}\left(H_{c}=1\right)$ & $-\operatorname{Ref} .[23]$ \\
\hline 1 & 1 & -1 & $1 s_{\frac{1}{2}}$ & -3.832037742 & -3.83216277 & -3.832237715 & -0.997911186 \\
2 & 1 & -2 & $1 p_{\frac{3}{2}}$ & -3.831636629 & -3.831862446 & -3.832037742 & -0.998123830 \\
3 & 1 & -3 & $1 d_{\frac{5}{2}}$ & -3.831032046 & -3.831359962 & -3.831636629 & -0.998420879 \\
4 & 1 & -4 & $1 f_{\frac{7}{2}}$ & -3.830220499 & -3.830652406 & -3.831032046 & -0.998776316 \\
1 & 2 & -1 & $2 s_{\frac{1}{2}}$ & -3.849079161 & -3.849180112 & -3.849240608 & -0.985981641 \\
2 & 2 & -2 & $2 p_{\frac{3}{2}}$ & -3.84875508 & -3.848937571 & -3.849079161 & -0.986559508 \\
3 & 2 & -3 & $2 d_{\frac{5}{2}}$ & -3.848265994 & -3.848531357 & -3.84875508 & -0.987401469 \\
4 & 2 & -4 & $2 f_{\frac{7}{2}}$ & -3.847608351 & -3.84795851 & -3.848265994 & -0.988478166 \\
1 & 1 & 2 & $0 d_{\frac{3}{2}}$ & -3.832037742 & -3.831862446 & -3.831636629 & -0.997911186 \\
2 & 1 & 3 & $0 f_{\frac{5}{2}}$ & -3.831636629 & -3.831359962 & -3.831032046 & -0.99812383 \\
3 & 1 & 4 & $0 g_{\frac{7}{2}}$ & -3.831032046 & -3.830652406 & -3.830220499 & -0.998420879 \\
4 & 1 & 5 & $0 h_{\frac{9}{2}}$ & -3.830220499 & -3.829735708 & -3.829197342 & -0.998776316 \\
1 & 2 & 2 & $1 d_{\frac{3}{2}}$ & -3.849079161 & -3.848937571 & -3.84875508 & -0.985981641 \\
2 & 2 & 3 & $1 f_{\frac{5}{2}}$ & -3.84875508 & -3.848531357 & -3.848265994 & -0.986559508 \\
3 & 2 & 4 & $1 g_{\frac{7}{2}}$ & -3.848265994 & -3.84795851 & -3.847608351 & -0.987401469 \\
4 & 2 & 5 & $1 h_{\frac{9}{2}}$ & -3.847608351 & -3.847214889 & -3.846777426 & -0.988478166 \\
\hline \hline
\end{tabular}

In Fig. 2, is plotted the energy vs. $\zeta$ for both pseudospin and spin symmetry limits for the Schioberg and general Manning-Rosen potentials respectively. In Fig. 3, are presented the effects of the tensor interaction on the bound states in view of the pseudospin and spin symmetry limits respectively. The figure shows that the magnitude of the energy 
difference between the degenerate states increases with the increase in $H_{c}$. Fig. 4 shows the effect of the Schioberg potential on the bound states in view of the pseudospin and spin symmetry limits respectively, and we can see that although bound states obtained in view of spin symmetry become more bounded with increasing $D^{s}$, they become less bounded in the pseudospin symmetry limit. Also, Figs. 5 and 6 show the energy for different values of $C_{p s}, C_{s}$ and $\sigma$ respectively.

Table 2. Energies in the Spin Symmetry Limit for $\zeta=0.05, M=1 \mathrm{fm}^{-1}, A^{s}=8$, $B^{s}=-8, C^{s}=-4.5, C_{s}=0, D^{s}=2, \sigma=-0.2$.

\begin{tabular}{|c|c|c|c|c|c|c|c|}
\hline$n$ & $\ell$ & $\kappa$ & $(\ell, j)$ & $E_{n \kappa}^{s}\left(H_{c}=0\right)$ & $E_{n \kappa}^{s}\left(H_{c}=0.5\right)$ & $E_{n \kappa}^{s}\left(H_{c}=1\right)$ & $\begin{array}{c}E_{n \kappa}^{s}\left(H_{c}=0 D^{s}=0\right) \\
- \text { Ref. }[23]\end{array}$ \\
\hline 0 & 0 & -1 & $0 S_{\frac{1}{2}}$ & 3.688759870 & 3.688808653 & 3.68875987 & 0.9592066538 \\
\hline 1 & 0 & -1 & $1 S_{\frac{1}{2}}^{2}$ & 3.723262967 & 3.723307088 & 3.723262967 & 0.9820439782 \\
\hline 2 & 0 & -1 & $2 S_{\frac{1}{2}}^{2}$ & 3.754434653 & 3.75447417 & 3.754434653 & 0.9957372014 \\
\hline 3 & 0 & -1 & $3 S_{\frac{1}{2}}^{2}$ & 3.782273862 & 3.782308806 & 3.782273862 & 0.9999778770 \\
\hline 0 & 1 & -2 & $0 P_{\frac{3}{2}}^{2}$ & 3.688369394 & 3.688613485 & 3.68875987 & 0.9587443558 \\
\hline 1 & 1 & -2 & $1 P_{\frac{3}{2}}^{2}$ & 3.722909793 & 3.72313057 & 3.723262967 & 0.9817364811 \\
\hline 2 & 1 & -2 & $2 P_{\frac{3}{2}}^{2}$ & 3.754118309 & 3.754316067 & 3.754434653 & 0.9955850262 \\
\hline 3 & 1 & -2 & $3 P_{\frac{3}{2}}^{2}$ & 3.781994111 & 3.782168998 & 3.782273862 & 0.9999875858 \\
\hline 0 & 2 & -3 & $o d_{\frac{5}{2}}^{2}$ & 3.687587311 & 3.688027418 & 3.688369394 & 0.9578120830 \\
\hline 1 & 2 & -3 & $1 d_{\frac{5}{2}}^{2}$ & 3.722202332 & 3.722600461 & 3.722909793 & 0.9811139378 \\
\hline 2 & 2 & -3 & $2 d_{\frac{5}{2}}^{2}$ & 3.753484519 & 3.753841206 & 3.754118309 & 0.9952729099 \\
\hline 3 & 2 & -3 & $3 d_{\frac{5}{2}}^{2}$ & 3.781433507 & 3.781749027 & 3.781994111 & 0.9999986412 \\
\hline 0 & 3 & -4 & $0 f_{\frac{7}{2}}^{2}$ & 3.686411362 & 3.687048756 & 3.687587311 & 0.9563944635 \\
\hline 1 & 3 & -4 & $1 f_{\frac{7}{2}}^{2}$ & 3.721138364 & 3.721715094 & 3.722202332 & 0.9801612652 \\
\hline 2 & 3 & -4 & $2 f_{\frac{7}{2}}^{2}$ & 3.752531081 & 3.753047937 & 3.753484519 & 0.9947853697 \\
\hline 3 & 3 & -4 & $3 f_{\frac{9}{2}}^{2}$ & 3.780589853 & 3.781047242 & 3.781433507 & 0.9999944089 \\
\hline 0 & 1 & 1 & $0 P_{\frac{1}{2}}$ & 3.688369394 & 3.688027418 & 3.687587311 & 0.9587443558 \\
\hline 1 & 1 & 1 & $1 P_{\frac{1}{2}}^{2}$ & 3.722909793 & 3.722600461 & 3.722202332 & 0.9817364811 \\
\hline 2 & 1 & 1 & $2 P_{\frac{1}{2}}^{2}$ & 3.754118309 & 3.753841206 & 3.753484519 & 0.9955850262 \\
\hline 3 & 1 & 1 & $3 P_{\frac{1}{2}}$ & 3.781994111 & 3.781749027 & 3.781433507 & 0.9999875858 \\
\hline 0 & 2 & 2 & $0 d_{\frac{3}{2}}^{2}$ & 3.687587311 & 3.687048756 & 3.686411362 & 0.9578120830 \\
\hline 1 & 2 & 2 & $1 d_{\frac{3}{2}}$ & 3.722202332 & 3.721715094 & 3.721138364 & 0.9811139378 \\
\hline 2 & 2 & 2 & $2 d_{\frac{3}{2}}$ & 3.753484519 & 3.753047937 & 3.752531081 & 0.9952729099 \\
\hline 3 & 2 & 2 & $3 d_{\frac{3}{2}}$ & 3.781433507 & 3.781047242 & 3.780589853 & 0.9999986412 \\
\hline 0 & 3 & 3 & $o f_{\frac{5}{2}}$ & 3.686411362 & 3.685674672 & 3.684838153 & 0.9563944635 \\
\hline 1 & 3 & 3 & $1 f_{\frac{5}{2}}^{2}$ & 3.721138364 & 3.72047169 & 3.719714552 & 0.9801612652 \\
\hline 2 & 3 & 3 & $2 f_{\frac{5}{2}}^{2}$ & 3.752531081 & 3.751933505 & 3.751254693 & 0.9947853697 \\
\hline 3 & 3 & 3 & $3 f_{\frac{5}{2}}^{2}$ & 3.780589853 & 3.780060894 & 3.779459851 & 0.9999944089 \\
\hline
\end{tabular}




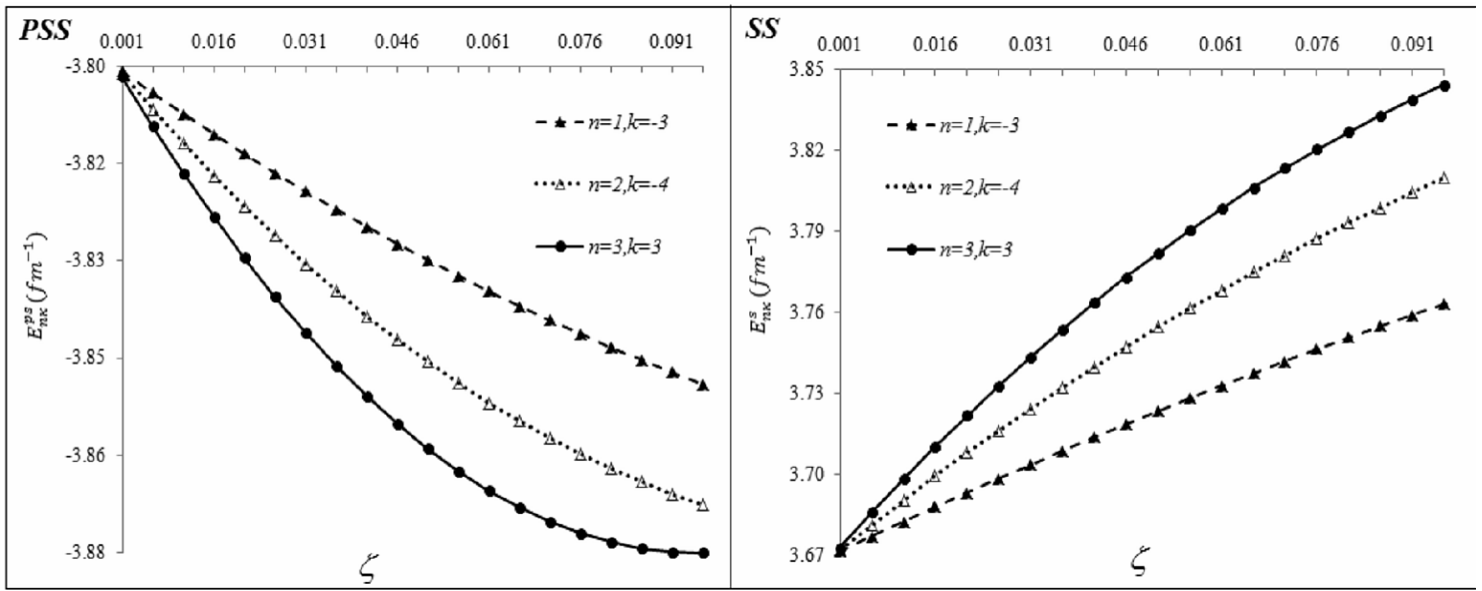

Figure 2. PSS: Energy vs. $\zeta$ for Pseudospin Symmetry Limit for $H_{c}=0.5, M=1 \mathrm{fm}^{-1}$, $A^{p s}=-8.75, B^{p s}=8.75, C^{p s}=4.5, C_{p s}=0, D^{p s}=-2, \sigma=-0.2$

SS: Energy vs. $\zeta$ for Spin Symmetry Limit for $H_{c}=0.5, M=1 \mathrm{fm}^{-1}, A^{s}=8$, $B^{s}=-8, C^{s}=-4.5, C_{s}=0, D^{s}=2, \sigma=-0.2$.
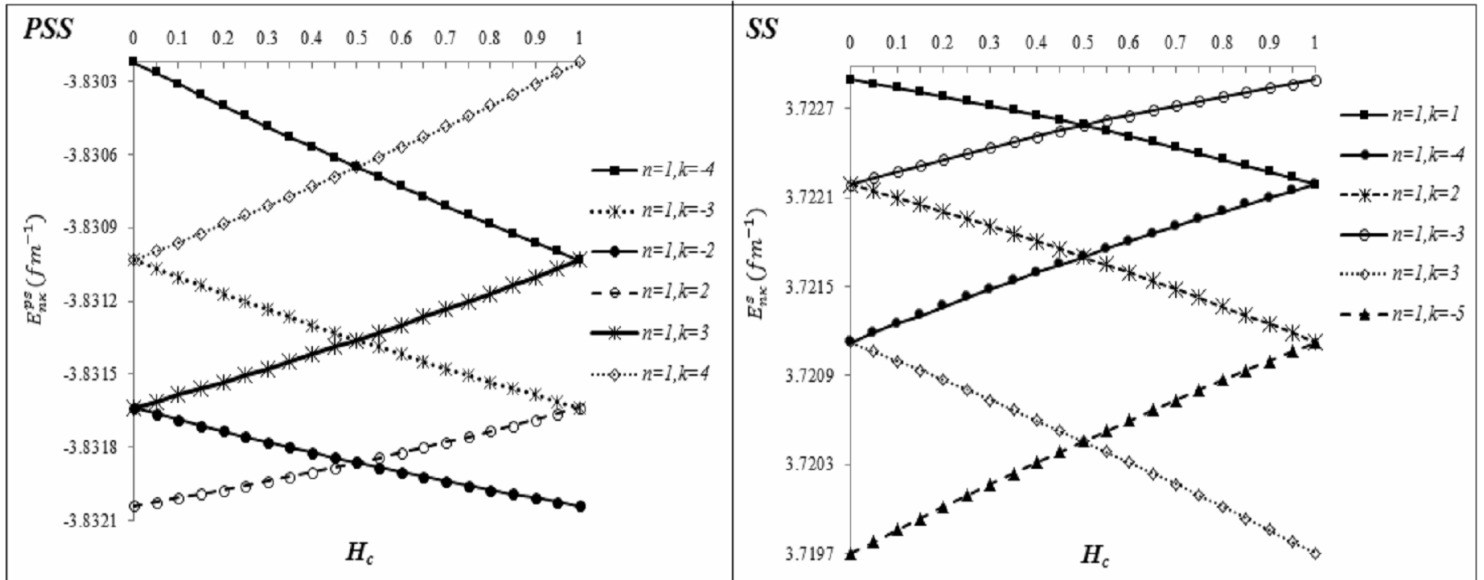

Figure 3. PSS: Energy vs. $H_{c}$ for Pseudospin Symmetry Limit for $\zeta=0.05, M=1 \mathrm{fm}^{-1}$, $A^{p s}=-8.75, B^{p s}=8.75, C^{p s}=4.5, C_{p s}=0, D^{p s}=-2, \sigma=-0.2$

SS: Energy vs. $H$ for Spin Symmetry Limit for $\zeta=0.05, M=1 \mathrm{fm}^{-1}, A^{s}=8$, $B^{s}=-8, C^{s}=-4.5, C_{s}=0, D^{s}=2, \sigma=-0.2$. 


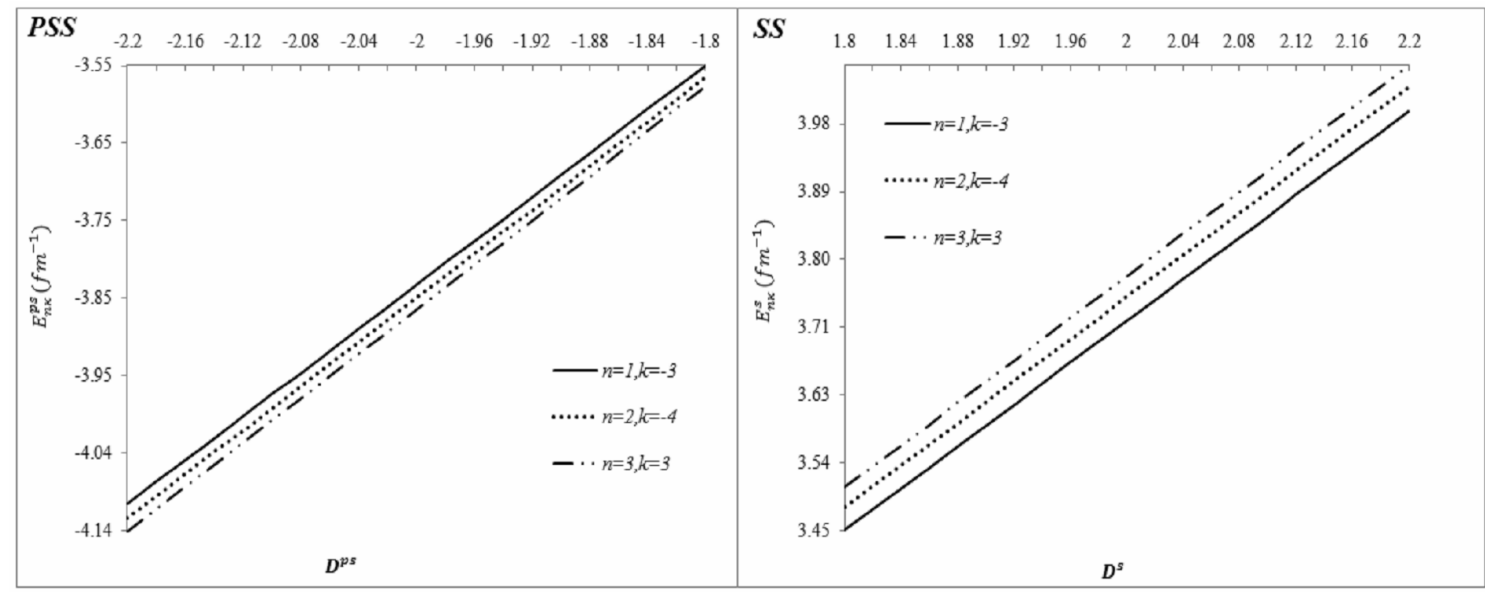

Figure 4. PSS: Energy vs. $D^{p s}$ for Pseudospin Symmetry Limit for $H_{c}=0.5, \zeta=0.05$, $M=1 \mathrm{fm}^{-1}, A^{p s}=-8.75, B^{p s}=8.75, C^{p s}=4.5, C_{p s}=0, D^{p s}=-2, \sigma=-0.2$ SS: Energy vs. $C_{s}$ for Spin Symmetry Limit for $H_{c}=0.5, \zeta=0.05, M=1 \mathrm{fm}^{-1}$, $A^{s}=8, B^{s}=-8, C^{s}=-4.5, C_{s}=0, D^{s}=2, \sigma=-0.2$.
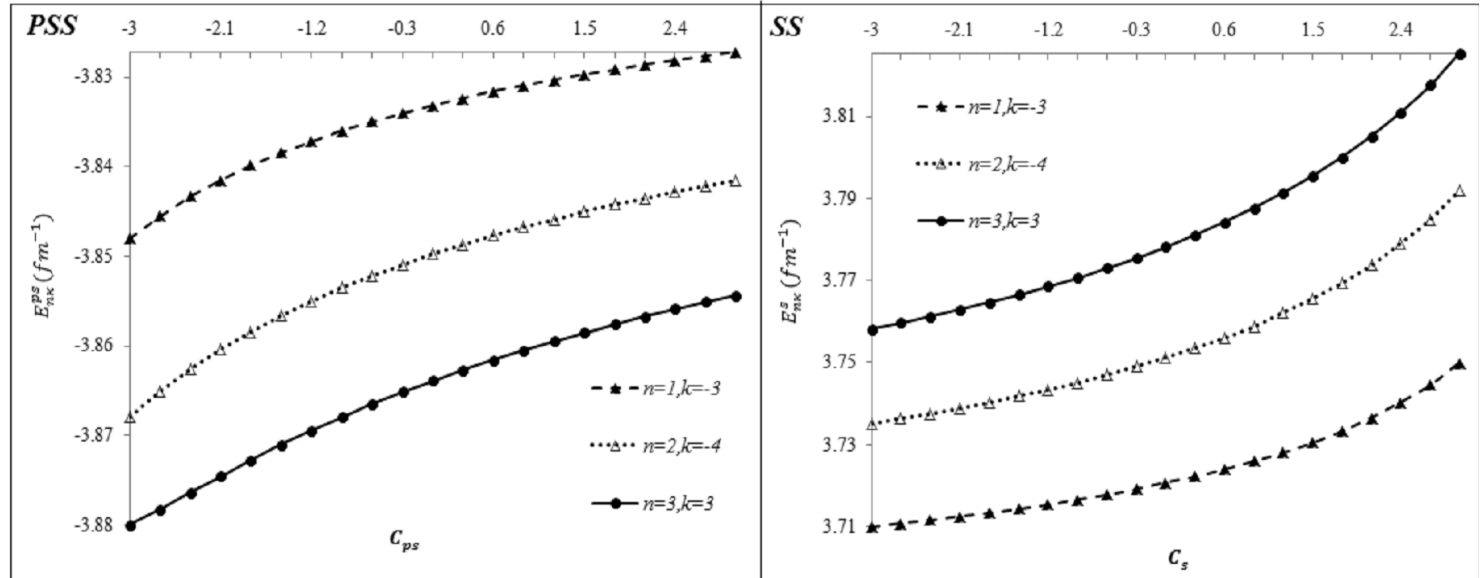

Figure 5. PSS: Energy vs. $C_{p s}$ for Pseudospin Symmetry Limit for $H_{c}=0.5, \zeta=0.05$, $M=1 \mathrm{fm}^{-1}, A^{p s}=-8.75, B^{p s}=8.75, C^{p s}=4.5, D^{p s}=-2, \sigma=-0.2$

SS: Energy vs. $C_{s}$ for Spin Symmetry Limit for $H_{c}=0.5, \zeta=0.05, M=1 \mathrm{fm}^{-1}$, $A^{s}=8, B^{s}=-8, C^{s}=-4.5, D^{s}=2, \sigma=-0.2$. 


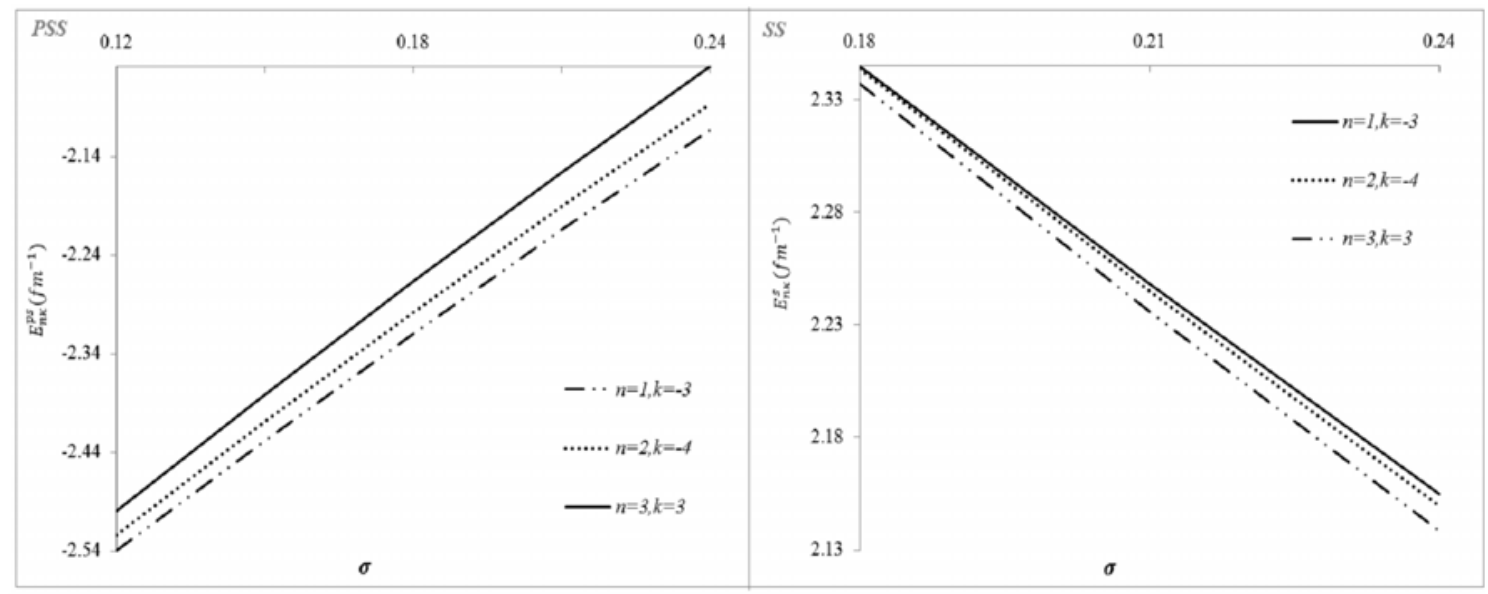

Figure 6. PSS: Energy vs. $\sigma$ for Pseudospin Symmetry Limit for $H_{c}=0.5, \zeta=0.05$, $M=1 \mathrm{fm}^{-1}, A^{p s}=-8.75, B^{p s}=8.75, C^{p s}=4.5, C_{p s}=0, D^{p s}=-2, \sigma=-0.2$ SS: Energy vs. $\sigma$ for Spin Symmetry Limit for $H_{c}=0.5, \zeta=0.05, M=1 \mathrm{fm}^{-1}$, $A^{s}=8, B^{s}=-8, C^{s}=-4.5, C_{s}=0, D^{s}=2, \sigma=-0.2$.

\section{Conclussion}

In the present paper we studied the approximate bound state solutions of the Dirac equation with combined Schioberg and general Manning-Rosen potentials by using the powerful SUSYQM techniques. We have obtained the energy levels in a closed form and the corresponding wave functions expressed in terms of the Jacobi polynomials for this potential model including the Coulomb-like tensor interaction within the spin and pseudospin symmetry limits. The computed numerical results show that the presence of this novel generalized tensor terms remove the degeneracies between two states in the spin and pseudospin doublets. The results of this work are more general compared to those reported in [23].

\section{References}

[1] F. Cooper, A. Khare and U. Sukhatme, Phys. Rep. 251, 267 (1995).

[2] G. Junker, Supersymmetric Methods in Quantum and Statistical Physics, (SpringerVerlag Berlin, 1996).

[3] G. F. Wei and S. H. Dong, Euro. Phys. Lett. 87, 4004 (2009).

[4] H. Hassanabadi, E. Maghsoodi and S.Zarrinkamar, Euro. Phys. J. Plus 127, 31 (2012).

[5] H. Hassanabadi, E. Maghsoodi, S. Zarrinkamar and H. Rahimov, Mod. Phys .Lett.A 26, 2703 (2011).

[6] A. N. Ikot, E. Maghsoodi, S. Zarrinkamar and H. Hassanabadi, Few-Body Syst. Doi: 10.1007/S00601-013-0701-6. 
[7] A. N. Ikot, O. A. Awoga, A. D. Antia, H. Hassanabadi and E. Maghsoodi, Few-Body Syst. Doi: 10.1007/s00601-013-0706-1.

[8] H. Hassanbadi and Z. Molacc, Chin. Phys. B 21, 120304 (2012).

[9] H. Hassanbadi, E. Maghsoodi, A. N. Ikot and S. Zarrinkmar, Appl. Math \& Compt. 219, 9388 (2013).

[10] M. Hamzavi, S. M. Ikhdair and B. I.Ita, Phys. Scr. 85, 045009 (2012).

[11] A. F. Nikiforov and V. B. Uvarov, Special Functions of Mathematical Physics (Birkhauser, Basel, 1988).

[12] H. Cifti, R. L. Hall and N. Saad, J. Phys. A 3611807 (2003).

[13] C. Rasinariu, Fortschr. Phys. 61, 4 (2013).

[14] E. Maghsoodi, H. Hassanabadi and O. Aydogdu, Phys .Scr. 86, 015005 (2012).

[15] S. H. Dong, Factorization Method in Quantum Mechanics (Springer, Dordreht, 2007).

[16] H. Taseli, Int. J. Quant. Chem. 63, 949 (1997).

[17] J. N. Ginocchio, Phys. Rev. C 69, 034318 (2004).

[18] J. N. Ginocchio, Phys. Rev. Lett. 78, 436 (1997).

[19] P. R. Page, T. Goldman and J. N. Ginocchio, Phys. Rev. Lett. 66, 204 (2001).

[20] J. N. Ginocchio, Phys. Rep. 414, 165 (2005).

[21] O. Aydogdu, E. Maghsoodi and H. Hassanabadi, Chin. Phys. B 22, 010302 (2013).

[22] S. M.Ikhdair and M. Hamzavi, Few-Body Syst. Doi: 10.1007/s00601-012-0475-2.

[23] H. Hassanabadi, E. Maghsoodi, S. Zarrinkamar and H. Rahimov, Can. J. Phys. 90, 633 (2012).

[24] A. N. Ikot, E. Maghsoodi, A. D.Antia, S. Zarrinkamar and H. Hassanabadi, Can. J. Phys. 91, 560 (2013).

[25] S. M. Ikhdair and R. Sever, Appl. Math. \& Comp. 216, 911 (2010).

[26] H. Hassanabadi, E. Maghsoodi and S. Zarrinkamar, Commun. Theor. Phys. 58, 807 (2012).

[27] A. N. Ikot, H. Hassanabadi, B. H. Yazarloo and S. Zarrinkamar (accepted to appear in Int. J. Mod. Phys. E).

[28] M. F. Manning and Rosen, Phys. Rev. 44, 953 (1933). 
[29] D. Schioberg, Mol. Phys. 59, 1123 (1986).

[30] R. L. Greene and C. Aldrich, Phys. Rev. A 44, 2363 (1976). 